\title{
La enseñanza por medio del juego para un mejor aprendizaje
}

Olga Rocío Morales Beltrán

Corporación Universitaria Minuto de Dios, Ceres-Zipaquirá

Olguitam1431@gmail.com

Zabdy Rocío Urrego Martínez²

Corporación Universitaria Minuto de Dios, Ceres-Zipaquirá

zabdyurrego.zu@gmail.com

1 Artículo producto del semillero de investigación de pedagogía infantil "UR3 UNIMINUTO Reduce, Recicle y Reutiliza".

2 Estudiantes de Licenciatura en Pedagogía Infantil de la Corporación Universitaria Minuto de Dios UNIMINUTO, Ceres-Zipaquirá. 


\title{
La enseñanza por medio del juego para un mejor aprendizaje
}

\section{Resumen}

El artículo parte de un proceso de investigación cualitativa, con una metodología de acción-participación, con el cual se buscó generar un espacio de recreación adecuado para los infantes, desarrollando estrategias pedagógicas para la enseñanza por medio del juego. Se implementó un proyecto ambiental sostenible que les brindó a ellos la oportunidad y la experiencia significativa de conocer más a fondo los problemas ambientales que se encuentran a su alrededor, particularmente a los alrededores del Colegio Campestre Colombo Británico, ubicado en el sector La Paz del municipio de Zipaquirá-Cundinamarca. Como punto de partida se apoyó un proceso de implementación del proyecto PRAE — proyectos ambientales escolares- de la institución mediante el desarrollo de actividades lúdico-pedagógicas para que los estudiantes conocieran las problemáticas ambientales, estableciendo como finalidad la creación e implementación del parque ecológico y la cartilla con el paso a paso para su construcción, la cual lleva por nombre: Elaboración, uso y funcionalidad pedagógica de las atracciones de un parque ecológico infantil, fuente de innovación estratégica para que la enseñanza se logre por medio del juego y obtener así un mejor aprendizaje. El parque ecológico fue construido a raíz de una necesidad, con intervención de los practicantes, y se involucró en el proceso a diferentes comunidades educativas tales como estudiantes del grado de preescolar, padres de familia docentes y administrativos.

Palabras clave: Juego, aprendizaje, enseñanza, parque ecológico, ambiente, educación, estrategia, 3R - reducir, reciclar, reutilizar-.

\section{Teaching Through Games for Better Learning}

\begin{abstract}
A qualitative-based investigation process was used in the present research, which includes an actionparticipation methodology. This methodology allowed the opening of an adequate leisure space for children in which pedagogical strategies were developed in order to explore game-based teaching. The implementation of a sustainable environment related project provided children with the meaningful experience of exploring in depth the environmental issues that surround them, particularly, related to Colegio Campestre Colombo Británico, in La Paz, Zipaquirá-Cundinamarca municipality. As a starting point, the implementation of PRAE -Proyectos ambientales escolares (School environment-related projects)- was supported by the development of ludic-pedagogical activities that help students explore environmental issues. The ultimate goal of the project was the creation and implementation of an eco-park and a booklet with information related to its design and construction. The title of the guide is: Elaboration, use and pedagogical functionality of the attractions of a children's ecological park. The whole project works as a strategic way to achieve game-based teaching for a better learning. The eco-park was built as a result of a necessity in the community. Practicants of the project, educational communities, such as preschool students, parents, teachers and administrative stuff participated in this project.
\end{abstract}

Keyords: Game, Learning, Teaching, Eco-Park, Environment, Education, Strategy, 3R -Reduce, Recycle, Reuse-.

\section{O ensino por meio do jogo para uma melhor aprendizagem}

\section{Resumo}

O artigo parte de um processo de investigação qualitativa, com uma metodologia de ação-participação, com o qual se procurou gerar um espaço de recreação adequado para os infantes, desenvolvendo estratégias pedagógicas para o ensino por meio do jogo. Implementou-se um projeto ambiental sustentável que lhes brindou a eles a oportunidade e a experiência significativa de conhecer mais a fundo os problemas ambientais que se encontram a seu ao redor, particularmente aos arredores do Colégio Campestre Colombo Britânico, localizado no setor La Paz do município de Zipaquirá-Cundinamarca. Como ponto de partida se apoiou um processo de implementação do projeto PRAE - projetos ambientais escolares- da instituição mediante o desenvolvimento de atividades lúdico-pedagógicas para que os estudantes conhecessem as problemáticas ambientais, estabelecendo como finalidade a criação e implementação do parque ecológico e a cartilla com o passo a passo para sua construção, a qual leva por nome: Elaboração, uso e funcionalidade pedagógica das atrações de um parque ecológico infantil, fonte de inovação estratégica para que o ensino se consiga por 
médio do jogo e obter assim uma melhor aprendizagem. O parque ecológico foi construído a raiz de uma necessidade, com intervenção dos praticantes, e envolveu-se no processo a diferentes comunidades educativas tais como estudantes do grau de preescolar, pais de família docentes e administrativos.

Palavras chave: Jogo, aprendizagem, ensino, parque ecológico, ambiente, educação, estratégia, 3R —reduzir, reciclar, reutilizar-. 


\section{Introducción}

El juego se ha convertido en parte esencial para el desarrollo del ser humano debido a que en él se establecen vínculos sociales, se canalizan energías y se adquieren conocimientos a través de sus experiencias, lo cual propicia la generación de un aprendizaje significativo. A primera vista se puede comprender que jugar es una estrategia para que los niños exploren y descubran el mundo por sí mismos. En palabras de Molina (2016):

El juego es un hecho motriz implícito en la práctica habitual del niño. Se considera el mejor medio educativo para favorecer el aprendizaje, fortaleciéndose con él todo el desarrollo físico y psicomotor, el desarrollo intelectual, y el socio-afectivo, normalmente requieren del uso mental o físico, y a menudo de ambos (p.1).

Por consiguiente, establecer un vínculo del juego con la enseñanza no es imposible, ya que durante el desarrollo de actividades lúdicas en las instituciones educativas se permite que los educandos desarrollen habilidades y destrezas, fortalecidas dentro del progreso del juego, adquiriendo así mayor comodidad y seguridad a la hora de comprender y adquirir un nuevo conocimiento durante el desarrollo de su vida escolar, lo que hace que su estadía sea cómoda y armoniosa, no aburrida ni tediosa.

Conviene, empero, advertir que se han generado controversias acerca del juego en la escuela, tal como se menciona en la obra El juego en la educación infantil: «el juego y el aprendizaje han estado siempre en la esencia de todos los debates, discusiones y propuestas que giran alrededor de la educación infantil» (Harf Rurt et al., 2008, p. 5). Los autores de esta obra, que es un conjunto de discusiones sobre la cotidianidad del juego, confrontan las situaciones que se viven en el jardín infantil ${ }^{3}$, buscando contribuir al rol docente y ampliando el valor del juego en sus diferentes clases. En primer lugar, se habla sobre la relación juego-aprendizaje y se menciona que para algunos estos son aliados y para otros son totalmente opuestos: «jugar para aprender, jugar y luego aprender, jugar no es aprender» (Harf Rurt et al., 2008, p. 5). El texto confronta muchos de los conceptos que se tienen sobre el juego y a su vez genera una serie de interrogantes que hacen que los autores se detengan a analizar y repensar lo que están haciendo. La obra brinda la oportunidad, por medio de ejemplos y situaciones cotidianas, de enfrentarse a una realidad que está latente en el aula de clase y retira esa loca idea según la cual el juego no es parte del trabajo o función del docente, de modo que:

3 Algunos de los temas que tratan los autores: si este no es el juego: ¿el juego dónde está? Preguntas y no respuestas. Jugar en la escuela: un diálogo que recién comienza. La espontaneidad en el juego y el movimiento en la educación inicial. Pequeños titiriteros y la kermesse: con el juego en la calle. 
Si el sentido de la escuela es la enseñanza y la actividad central de la vida del niño es el juego, es hora de dejar de lado los estereotipos y esquemas rígidos y comprender que es lícito enseñar a través del juego (Harf Rurt et al., 2008, p. 5).

Por otro lado, los autores infieren y establecen que el objetivo principal de asistir a las instituciones educativas es que los estudiantes adquieran conocimientos sin interesarles cuáles sean los medios y las estrategias para lograrlo; por tal razón es posible intuir que el aprendizaje ocurre por medio de la práctica o de otras formas de experiencia en las que «el ser humano interactúa y tiene la disposición de aprender de verdad sólo aquello a lo que le encuentra sentido o lógica» (Sitio Web EcuRed, 2016). Con esto se afirma que, el ser humano tiene disposición de aprender lo que para él es significativo pues llama su atención y curiosidad, contrario a lo que sucede cuando es obligado a entender e incorporar información a la cual no le encuentra importancia y que, por tal razón, tiende a rechazar.

De manera que se busca dar fin al paradigma según el cual no es posible enseñar por medio del juego, puesto que no se logra un buen aprendizaje, aunque resulte ser todo lo contrario: al jugar, el estudiante retroalimenta el contenido orientado por el docente, comprendiéndolo por sí mismo y llevándolo a la práctica, ya que lo convierte en aprendizaje significativo. Es allí donde la intervención del docente tiene gran importancia, pues éste tiene la responsabilidad de captar y llamar la atención del niño para que sienta curiosidad y ganas de aprender por medio del manejo de las metodologías y herramientas que presenta el juego. El juego, en fin, es un instrumento útil, eficaz, que ayuda a comprender, adquirir y profundizar nuevos conocimientos, como fuente para un mejor aprendizaje.

Dado lo anterior, el objetivo general de la investigación fue el de crear una propuesta pedagógica que involucrara cuatro aspectos importantes dentro de la comunidad: enseñanza, juego, aprendizaje y cuidado del medio ambiente. Por tal razón, en el Colegio Campestre Colombo Británico del municipio de ZipaquiráCundinamarca se desarrolló un proyecto lúdico, pedagógico y ambiental a través de estrategias en las que los estudiantes son los protagonistas dentro de las actividades y programas. Es así como nace la iniciativa de realizar un proyecto ambiental en el que, mediante sus experiencias y vivencias, los estudiantes adquieran nuevos conceptos y conocimientos frente a las problemáticas ambientales que se encuentran a su alrededor, para de ese modo contribuir a su disminución y generar transformación e impacto social dentro de la comunidad.

Para tal fin se propuso la creación y elaboración de un parque ecológico y la cartilla con el paso a paso para su construcción, la cual, como se dijo anteriormente, lleva por nombre: Elaboración, uso y funcionalidad pedagógica de las atracciones de un parque ecológico infantil; un centro de aprovechamiento lúdico-recreativo estratégico en el ámbito pedagógico para sus estudiantes, pues cada una de sus 
atracciones tiene una funcionalidad pedagógica, académica y educativa. Para su construcción se contó con la participación de las practicantes responsables de la investigación, estudiantes del grado de preescolar — prekinder, kínder y transición-, padres de familia, docentes y administrativos.

De acuerdo con el proyecto se utilizó material abandonado por problemas ambientales y se establecieron convenios con establecimientos cercanos a la institución para la recolección de materiales —neumáticos, madera, plástico, entre otros- que han sido desechados, con el fin de generar un nuevo uso y prolongar su vida útil mediante las 3R: reciclar, reducir y reutilizar.

Se pretende, finalmente, que se sigan implementando proyectos ambientales escolares —PRAE - en pro de la educación que causen impacto — transformación social- y que involucren recreación, lúdica, pedagogía, enseñanza, y aprendizaje para los educandos.

\section{Marco teórico}

El juego es parte fundamental en el desarrollo de los niños y niñas, tal y como señala María Isabel Benítez Murillo en su artículo "El juego como herramienta de aprendizaje" (2009): «el juego es de gran importancia para el sano desarrollo de la personalidad infantil» (p. 2). Allí se resalta que los niños se comunican y se expresan por medio del juego y que, aunque existan diferentes culturas, creencias, niveles sociales, económicos y se empleen diferentes juegos, todos han tenido la experiencia de jugar y divertirse. Asimismo, el juego lo han implementado en las instituciones educativas para lograr que los educandos obtengan un aprendizaje significativo, lo que deja de lado a la educación tradicional, en la cual los niños obtenían un aprendizaje memorístico y repetitivo. Estas metodologías eran de enseñanza extrema debido a que los estudiantes no podían opinar y mucho menos descubrir nuevos conocimientos a través de sus experiencias y vivencias, que se establecen de manera autónoma.

Al respecto, conviene decir que durante el desarrollo del juego los seres humanos desarrollan capacidades y habilidades físicas, sensoriales, mentales, psicomotrices y lógicas, fortaleciendo así su creatividad e imaginación. Llegados a este punto, se debe enfatizar que los juegos tradicionales han aportado por generaciones a la educación de los niños y niñas, pues actualmente, con las nuevas tendencias tecnológicas, se ha perdido el interés por crear y descubrir autónomamente, por establecer vínculos socioafectivos.

Aquí es necesario detenerse un momento y preguntarse: ¿hasta dónde ha llegado la iniciativa de determinar un vínculo entre la enseñanza, el juego y el aprendizaje 
en la educación y formación de los estudiantes? Lo anterior se considera toda vez que el juego es fuente de iniciativa ante un proceso de transformación social, dado que puede crear y establecer proyectos en los que se vincula el cuidado del medio ambiente y se desarrollan, de igual forma, estrategias lúdico-pedagógicas para el proceso educativo de los educandos.

Por lo anterior, se toma como referencia a las hermanas Agazzi — Rosa y Carolina Agazzi-, quienes son pioneras en la innovación de la educación infantil debido a la implementación de materiales de uso cotidiano no costosos como herramientas didácticas para el proceso educativo de sus estudiantes: «los principios de las Agazzi, están basados en el uso inteligente de las cosas, en la educación del lenguaje y de los sentidos a partir del contacto con la naturaleza y los objetos» (De la Cruz, 2012, p.16). Cabe resaltar que los implementos lúdicos o baratijas — como ellas lo llamaban- eran creados con el material que los niños y niñas ayudaban a recoger y que denominaban «materiales vivos».

Estos objetos están dotados de características de sencillez y claridad, que los hacen atractivos para los niños y estimulan el juego y la adquisición de conocimientos importantes como las formas, los tamaños, el volumen, es decir trabajando sus cualidades, clasificando, ordenando, agrupando, diferenciando (De la Cruz, 2012, p. 17).

De ahí que, es importante para captar la atención de los estudiantes implementar material lúdico y Ilamativo para que ellos quieran explorar, indagar y obtener nuevos conocimientos; materiales que sean un apoyo para las diferentes áreas o dimensiones que se estén trabajando y, de esa forma, los educandos obtengan aprendizajes significativos por medio de sus vivencias y experiencias.

Vigotsky enfatiza en que el juego es un «instrumento y recurso socio-cultural» (Como cita en Tripero, 2011, p. 1), lo cual quiere decir que el juego involucra a un grupo de varias personas que interactúan e intercambian ideas entre sí y, además, desarrollan actividades en las que establecen vínculos socioafectivos, se diseñan estrategias, acatando y respetando las reglas establecidas.

De otro lado, Vigotsky hace alusión a que: «El juego es una realidad cambiante y sobre todo impulsora del desarrollo mental del niño, concentrar la atención, memorizar y recordar se hace en el juego de manera consciente, divertida y sin ninguna dificultad» (como se cita en Tripero, 2011.). Esto ayuda a que el niño despeje su mente y retroalimente el contenido visto en clase de una manera diferente, pues se encuentra con la satisfacción de aprender por interés y no por presión e imposición.

Melanie Klein se refiere al juego como: «La mejor expresión del niño» (como se cita en Torres, 2002, p. 122), por ende, los niños y las niñas pueden expresar 
sus sentimientos, emociones y su punto de vista intercambiando y respetando ideas sin cohibirse; así, los niños desarrollan su personalidad y dejan el miedo a lo que los otros piensen de ellos, además ayuda a que los docentes detecten lo que está viviendo o sintiendo un estudiante frente a alguna problemática familiar o dentro del ambiente escolar, lo que permite que sea más autónomo e independiente.

Woods Winnicott considera que: «El juego [es] una actividad central de estos procesos — procesos que Ilevan a la construcción del ser humano-. Durante ese proceso se repiten, se olvidan, se retoman actividades lúdicas que favorecen la socialización del humano» (como se cita en Torres, 2002, p. 122). Vale resaltar que, de esta forma, los niños interactúan y socializan con los demás de una forma fácil y constituyen nuevas relaciones afectivas con sus compañeros y docentes, aprendiendo y transmitiendo algún conocimiento o experiencia propia. A su vez, Woods agrega que: "El jugar tiene un lugar y un tiempo, no se encuentra "adentro" ni tampoco está "afuera". Jugar es hacer» (2002, p.123). Con todo lo anterior, se puede afirmar que jugar no necesariamente se desarrolla en un lugar determinado, pues el aula de clase se puede modificar y ambientar para desarrollar la actividad establecida; de la misma manera, si ésta se desarrolla en un campo abierto, lo único que se debe hacer es darle libertad al niño de imaginar y crear: "Jugar es una terapia que los niños y adultos tienen la libertad de crear» (Como cita Torres, 2002).

Por otro lado, Jean Piaget argumenta que: «En el juego el niño es como si fuera una cabeza más alto de lo que en realidad es» (Torres, 2002, p. 124). Como es sabido, los niños se sienten seguros al desarrollar una actividad en la que el juego es parte importante en el proceso de enseñanza-aprendizaje, pues están cómodos al desarrollar la actividad pedagógica sin necesidad de tener que aprenderse algo de memoria o que les impongan algo que les disgusta o no han logrado comprender. También cabe recalcar que el niño opina, resuelve problemas complejos y participa de las diferentes actividades si se emplea el juego como metodología de enseñanzaaprendizaje.

Habría que agregar que en la etapa del preescolar el niño aprende a permanecer en el aula sin traumas, pues el problema reside cuando ingresa a la educación básica. En esta fase educativa el juego desaparece por completo y su permanencia es un poco más difícil dado que vienen aprendizajes un poco más complejos en los que el docente maneja metodologías más teóricas y, por ende, a los estudiantes se les dificulta un poco más. No obstante, a medida que pasa el tiempo, aunque ellos se van adaptando a sus nuevos mecanismos de aprendizaje, continúan extrañando un poco la forma aprendizaje-juego-enseñanza.

Como se mencionó anteriormente, las hermanas Agazzi han hecho un aporte significativo que guiará el proceso del proyecto pedagógico-ambiental porque la recursividad que manejaron frente a sus diferentes actividades pedagógicas 
ha producido un enfoque para el desarrollo del proyecto. Este proyecto ha sido orientado al manejo de los residuos sólidos, por lo que es importante mencionar de qué se tratan y la relevancia que tienen las tres erres ecológicas — $3 \mathrm{R}-$, reducir, reciclar y reutilizar:

Toda la población del mundo abarca alrededor de 7 mil millones de personas, las cuales están comprando y desechando desperdicios exactamente al mismo tiempo. La manufactura de tanta mercancía, utilizando los métodos actuales, resulta una carga muy pesada para el planeta en todos los aspectos (El Impulso.com, 2013).

La regla de las tres erres, también conocida como las tres erres de la ecología o simplemente $3 \mathrm{R}$ — reducir, reciclar y reutilizar-, es una propuesta sobre hábitos de consumo, popularizada por la organización ecologista Greenpeace, que pretende desarrollar hábitos generales responsables como el consumo responsable (El Impulso. com, 2013). Este concepto busca generar estrategias para el manejo y clasificación adecuada de los residuos sólidos, los cuales son desechados y causan contaminación ambiental, razón por la cual se busca transformar este material para prolongar su vida útil y lograr así disminuir en gran cantidad la contaminación generada.

Para forjar un cambio y obtener resultados, se deben establecer como consumidores hábitos y actitudes a la hora de desechar algún envase o producto y considerar el efecto que tienen sobre al medio ambiente, buscando la manera de resolverlos mediante el involucramiento y la orientación a la sociedad.

Por consiguiente, es necesario desarrollar proyectos pedagógicos en los que se dé a conocer a docentes, padres de familia y estudiantes los problemas ambientales que se están provocando debido a la poca cultura y conciencia que se tiene frente a los desechos generados y que, como seres humanos, arrojamos sin tener precaución o consideración sobre las consecuencias que trae para el medio y que se han extendido a todo el planeta Tierra, causando diversos riesgos.

Esta propuesta está proyectada a la creación de un impacto social en el que se establezcan estrategias para lograr disminuir la contaminación y en la que las instituciones educativas, tanto públicas como privadas, sean el ente más cercano para contrarrestar tal problemática, generando estrategias educativas ambientales para que los estudiantes, desde pequeños, tengan una visión amplia y se desarrolle en ellos conciencia y cultura ambiental. Aun así vale preguntarse: ¿hasta dónde debe llegar el problema ambiental para verdaderamente tener conciencia y empezar a cambiar?

En varias poblaciones de países y culturas diferentes se han empezado a desarrollar proyectos que buscan disminuir las problemáticas ambientales que se están creando en el planeta, por ejemplo, a raíz de los neumáticos que han sido abandonados en 
sectores públicos, en parques y en zonas verdes - los cuales causan problemas de salud-; para ello, se ha venido trabajando en la creación de diferentes productos, como los pisos decorativos de caucho, materas de neumáticos, y en la construcción de parques ecológicos como fuente de recreación, todo a base de las llantas que se han desechado y cuya materia prima ha tenido una transformación que logra disminuir los problemas ambientales y prolonga su utilidad.

A la hora de implementar la primera $\mathrm{R}$, que es reducir, se hace referencia a la eliminación de la cantidad de materiales destinados a un uso único; de igual forma, pretende evitar el uso de productos desechables, los cuales son el enemigo número uno del medio ambiente, por ejemplo, para reducir la contaminación ambiental se hace necesario cambiar las bolsas plásticas por bolsas de tela, que son reutilizables, debido a que cada bolsa plástica se demora por lo menos quinientos años en descomponerse por completo. Empero, a la hora de desechar algún envase o producto se debe tener en claro que hay personas o empresas que se encargan de transformar este material y prolongan de ese modo su vida útil, esto es, desarrollan en él nuevas utilidades. Es allí donde aparece la segunda $\mathrm{R}$, la cual consiste en reciclar materiales usados o desperdicios, sometiéndolos a un proceso de transformación para que sean nuevamente utilizados. Tal transformación se puede propiciar en materiales como el papel, el vidrio y el plástico, lo que genera la creación y construcción de nuevos productos. Por último, se encuentra la tercera $\mathrm{R}$, reutilizar, que radica en utilizar algo que tenía otra funcionalidad para extender su vida útil, por ejemplo, los envases plásticos pueden tener una evolución y crearse, con base en ellos, portalápices, materas, joyeros, flores, entre otros accesorios —en vez de botar y comprar, mejor crear-, lo cual beneficia y ayuda al medio ambiente.

\section{Metodología}

Fue una investigación de carácter cualitativo, de acción-participación, puesto que:

[...] las técnicas cualitativas son útiles en sí mismas con el propósito de encontrar o interpretar las respuestas de las personas o comunidades intentando identificar el significado sociocultural que un evento dado puede tener en una comunidad, utilizando técnicas de observación, además investiga el cómo y el por qué se tomó una decisión (Enciso, 2013).

Desde este punto de partida, se hace un diagnóstico de observación que permitió establecer cuáles eran las problemáticas dentro de la comunidad y cuáles serían las posibles soluciones; para ello se realizó una indagación acerca de los diferentes proyectos que se han efectuado a nivel nacional e internacional para que guíen el proceso que se emprendió. Se pretendió, pues, dar respuesta a la siguiente pregunta de investigación: ¿cómo el juego interfiere en el proceso de enseñanza y aprendizaje desde la educación ambiental? 
Por ende, fue necesario realizar una subdivisión de cada uno de los elementos a trabajar mediante un análisis de categorías. Producto de ello se extrajo la categoría «la enseñanza por medio del juego para un mejor aprendizaje», y se obtuvo como subcategorías: educación, cultura y pedagogía. Posteriormente, cada una se unificó de acuerdo a los intereses de la investigación.

La primera subcategoría es educación. Por medio de las experiencias y vivencias, los estudiantes adquieren aprendizajes significativos, lo cual causa un impacto social y de sostenibilidad ambiental; la segunda subcategoría, cultura, fomenta interacciones socioculturales para la adaptación al medio, desarrollando procesos continuos de aprendizaje —enseñar-aprender-; y la tercera, pedagogía, es el «lugar» donde se genera impacto dentro de la comunidad —construcción-.

Para llevar a cabo la construcción del análisis de categorías fue necesario realizar una lectura profunda de los documentos seleccionados, que serían parte importante para el desarrollo de la investigación, y se elaboró, por tanto, un Resumen Analítico en Educación — RAE- de cada uno de los documentos.

Este proyecto de investigación, como ya se dijo, partió de un enfoque de acciónparticipación, en el cual el investigador no sólo requiere conocer una determinada realidad o problemática dentro de la comunidad a trabajar, sino que busca resolverla, dándole participación activa al grupo dentro de cada una de las etapas de desarrollo del proyecto.

\section{Resultados}

Los resultados obtenidos a través de este proceso de investigación, desarrollado en el Colegio Campestre Colombo Británico en el municipio de Zipaquirá, son los siguientes: inicialmente se logró concientizar a estudiantes, docentes y administrativos sobre el uso adecuado de los residuos sólidos, orgánicos e inorgánicos, lo que respondió al problema de la falta de conciencia a la hora de desechar los residuos — primer resultado_- p por otro lado, se les enseñó a los estudiantes, por medio de talleres lúdicos, distintas maneras de reutilizar los residuos sólidos, quienes, además, crearon productos autosostenibles para su consiguiente reutilización segundo resultado- . Esto creó un gran interés en los estudiantes sobre el cuidado del medio ambiente y de ahí se partió para la creación de un grupo de monitores, encargados de orientar y dar uso adecuado a los residuos sólidos de la institución, el cual se denominó «Agentes ecológicos», un «minisemillero» fundado con el fin de mejorar la situación ambiental de la institución y fortalecer el proyecto PRAE de la misma, el cual sigue activo hasta este momento — tercer resultado, que forjó nuevos conocimientos en cada estudiante-. Por último, el resultado principal fue la creación e implementación de un parque ecológico donde cada atracción tiene un 
fin educativo que permite a los estudiantes aprender mientras se divierten: aprenden y refuerzan los conocimientos y conceptos adquiridos en su proceso educativo.

\section{Conclusiones}

A partir del proceso de investigación, desarrollado e implementado en el Colegio Campestre Colombo Británico, se logró la participación activa de estudiantes, docentes y administrativos, quienes fueron sensibilizados y concientizados sobre el cuidado del medio ambiente. Se propició el reciclaje, la reutilización y la reducción de los residuos sólidos por medio de talleres lúdicos que crearon estrategias pedagógicas y educativas para los estudiantes —enseñanza-aprendizaje-.

Vale señalar que la revisión documental fortalece el proceso de investigación, por ende, se crea el parque ecológico infantil con fines recreativos y pedagógicos. Se puede evidenciar que durante su uso y aplicación los estudiantes adquieren aprendizajes significativos, comprendiéndolos a través de sus vivencias y experiencias; el parque, pues, es una herramienta útil durante el proceso académico de los estudiantes.

Así las cosas, se realiza el artículo de divulgación: "La enseñanza por medio del juego para un mejor aprendizaje", y la cartilla con el paso a paso para su construcción, la cual lleva por nombre: "Elaboración, uso y funcionalidad pedagógica de las atracciones de un parque ecológico infantil", como una iniciativa de nuevos proyectos ambientales en pro de la educación.

Se cumplieron de esta manera los objetivos establecidos al inicio de la propuesta de investigación mencionada anteriormente y se consiguió éxito gracias a la aceptación obtenida de parte de toda la comunidad estudiantil, docente y administrativa. 


\section{Referencias}

Álvarez, V. (4 de agosto de 2011). Felicidad de miles de niños llega con material reciclado. Recuperado de http://www.eltiempo.com/archivo/documento/MAM-4730235

De la Cruz Ceinos, S. (2012). Pioneras en la educacion infantil: los modelos educativos Agazzi y Mcmillan versus modelo de E.I vigente español [trabajo de grado]. Valladolid: Universidad de Valladolid.

EcuRed. (7 de septiembre de 2016). Aprendizaje. Recuperado de https://www.ecured.cu/ Aprendizaje

El Impulso.com. (15 de Abril de 2013). Las tres erres ecológicas: reducir, reutilizar, reciclar. Recuperado de http://www.elimpulso.com/noticias/actualidad/las-tres-erres-ecologicasreducir-reutilizar-reciclar

Enciso, C. (8 de octubre de 2013). El Paradigma Hermenéutico, la invetigacion cualitativa. Recuperado de https://prezi.com/3idx6un3nezq/el-paradigma-hermeneutico-la-investigacioncualitativa/

Garcia, J., \& zais, B. (2012-2014). Diseño y Construcción de un parque infantil con elementos reutilizados en Kumbungu, Ghana. Recuperado de http://www.upv.es/entidades/CCD/ infoweb/ccd/info/U0676115.pdf

Harf, R., et.al. (2008). El juego en la educación infantil: crecer jugando y aprendiendo. Buenos Aires: Centro de Publicaciones Educativas y Material Didáctico.

Itaipu Binacional. (4 de septiembre de 2012). Construyen parque ecológico en Hernandarias. Recuperado de https://www.itaipu.gov.br/es/sala-de-prensa/noticia/construyen-parqueecologico-en-hernandarias

Malaver, C. (11 de julio de 2014). Llantas que dan felicidad a niños necesitados. Universitarios convierten neumáticos abandonados en parques para los menores. Recuperado de El Tiempo.com: http://www.eltiempo.com/bogota/universitarios-crean-parques-para-ninoscon-Ilantas/14238039

Molina, Raúl. (2016). El concepto de juego y su importancia dentro del ámbito educativo en escolares de 10 a 12 años. Educación Física y Deportes, Revista Digital, 21(221), 1-1.

Murillo, M. I. (16 de Marzo de 2009). El juego como herramienta de aprendizaje. Recuperado de http://www.csi-csif.es/andalucia/modules/mod_ense/revista/pdf/Numero_16/MARIA $\% 20 I S A B E L \_B E N I T E Z \_1 . p d f$

Revista Su Casa. (25 de marzo de 2013). Gira y da vueltas. Recuperado de http://www. revistasucasa.com/articulo/especiales/un-\%E2\%80\%98play\%E2\%80\%99-de-Ilantas

Serrano, M. (25 de Septiembre de 2015). Proyecto de adecuación de parques con el uso de llantas recicladas en la localidad de Teusaquillo de la ciudad de Bogotá [tesis de pregrado]. Bogotá: Universidad Nacional Abierta y a Distancia. 
Torres, C. M. (18 de enero de 2002). El juego como estrategia de aprendizaje en el aula. Mérida: Artículos, Pre-prints, Centro de Investigaciones para el Desarrollo Integral Sustentable (CIDIS).

Tripero, A. (5 de enero de 2011). Vigotsky y su teoría constructivista del juego. Recuperado de http://biblioteca.ucm.es/revcul/e-learning-innova/5/art382.php\#.VvGpoeLhDIV

Werner, D. (1990). Parques de juegos para todos los niños. En D. Werner, El niño campesino deshabilitado (pp. 415-426). Berkeley: Hesperian.

Recibido: 9 de octubre 2016

Aceptado: 16 de noviembre 2016

Cómo citar: Morales, O., y Urrego, Z. (2017). La enseñanza por medio del juego para un mejor aprendizaje. Praxis Pedagógica, 20, 123-136 